\title{
BOOKS REVIEWED
}

Tuberous Sclerosis Complex: From Basic Science to Clinical Phenotypes. 2003. Edited by Paolo Curatolo. Published by Mac Keith Press. 314 pages. C\$112 approx.

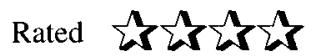

Tuberous Sclerosis Complex (TSC) is one of the most common neurocutaneous disorders. Since the initial description of "sclerose tubereuse" by Bourneville in 1880, there has been an explosion of new information about the disorder. Curatolo, himself, mentions in the book that approximately 500 papers are published each year in the field. Therefore, clinicians require regular updating.

This book, edited by Curatolo, a world authority on TSC, summarizes the wealth of information in a comprehensive, wellorganized, and easy to read fashion. Clinicians can refer to this single source for an instant update on a vast array of information, from clinical features to the basic neurosciences. The first part of the book provides historical background for the reader. Subsequent chapters discuss clinical advances, diagnostic criteria, clinical phenotypes, diagnostic imaging, and neuropsychological data. The final chapters shed light on the pathophysiology of the disease and new advances in the basic sciences regarding molecular and genetic data.

Various neurological aspects are covered, including neurological manifestations, epilepsy, intellectual and cognitive impairments, and autism. Current treatments are also discussed, including anti-epileptic drugs, and a brief mention of epilepsy surgery. There are also well written chapters summarizing current data on neuro-imaging and positron emission tomography (PET) scanning.

Tuberous Sclerosis Complex is a condition with multi-system involvement. The book is enriched by chapters written by authors from different disciplines to summarize relevant information. Chapters are written by those who are authorities in their respective fields, such as dermatologists, cardiologists, geneticists, and pathologists. This provides the neurologist with detailed information in areas outside their expertise. The text is enhanced by high quality photographs exemplifying clinical features and radiological scans.

Overall, the book covers various aspects of the condition. However, chapters are brief and only provide summaries. Therefore, if the reader requires thorough information, other references must be sought. Another drawback is that it was published in 2003. Since that time, there is a large amount of new information in the field, especially in the basic sciences. As there are more recent books on $\mathrm{TSC}$, it is no longer the most up to date reference.

This book provides a comprehensive summary of TSC. It also piques the reader's interest and discusses future developments and areas of research. Although no book is comprehensive due to continuous advancements, this text deserves a place on the clinician's shelf as an authoritative contemporary reference on TSC. It is also succinct so that a cover to cover read provides a quick update in current knowledge on TSC. However, this text needs to be supplemented by additional reading for up to date information due to its publication date of 2003 .

Anita Datta

Saskatoon, Saskatchewan, Canada

The Opiate Receptors. Second Edition. 2011. Edited by Gavril W. Pasternak. Published by Humana Press. 516 pages. $\mathrm{C} \$ 230$ approx.

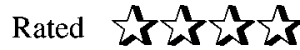

There have been major advances in opiate receptor pharmacology since the first edition of this book was published in 1988. The major goal of this second edition of The Opiate Receptors is to provide insight into the molecular and classical pharmacology of opiates and opioid peptides and to translate this into pharmacological actions. Gavril Pasternak, as the editor of both volumes and a world leader in opiate receptor pharmacology for four decades, does a masterful job of bringing all of this together.

The early chapters provide a historical perspective on the concept of multiple opiate receptors and lay the ground work for the molecular and functional characterization of the mu, delta and kappa receptors. Cloning of these receptors has been the major advance in allowing this work to move forward. The chapter on opioid receptor trafficking is especially illuminating in detailing the activation, inactivation and desensitization of opioid receptors as they cycle through the classical biosynthetic and endocytic pathways. For clinicians who manage pain, the discussion of molecular modulation of in vivo tolerance provides insights into the mechanisms of analgesic tolerance and potential approaches to reduce or eliminate this phenomenon. The final chapter on the genetics of opioid actions examines the current state of knowledge of opioid genetics and helps to explain the striking inter-individual variability in the therapeutic and side effect responses to opioid analgesics. For instance, who knew that kappa agonists such as pentazocine provide greater analgesia in female redheads compared to nonredheads!

This book will be a valuable resource for scientists working in the opiate field and for clinicians who are interested in the pharmacological actions of these agents. However, it is clear that much more work needs to be done if we are going to achieve the holy grail of providing powerful analgesics without significant side effects and addictive potential. 